\title{
Intermixing of motional currents in suspended CNT-FET based resonators
}

\section{Conference Paper}

\section{Author(s):}

Kumar, Lalit; Jenni, Laura Vera; Haluska, Miroslav; Roman, Cosmin; Hierold, Christofer

Publication date:

2018

Permanent link:

https://doi.org/10.3929/ethz-b-000331811

Rights / license:

In Copyright - Non-Commercial Use Permitted

Originally published in:

https://doi.org/10.1109/iedm.2018.8614660

\section{Funding acknowledgement:}

153292 - Q-factor enhancement in resonating CNTs (SNF) 


\title{
Intermixing of motional currents in suspended CNT-FET based resonators
}

\author{
Lalit Kumar, Laura Vera Jenni, Miroslav Haluska, Cosmin Roman and Christofer Hierold \\ Micro and -Nanosystems, Department of Mechanical and Process Engineering, ETH Zurich, Switzerland \\ Email: lalit.kumar@micro.mavt.ethz.ch
}

\begin{abstract}
Here, we report the intermixing of piezoresistive and conduction modulation current in a carbon nanotube field effect transistor (CNT-FET) based resonator. We show that due to static displacement of the nanotube, as a result of electrostatic actuation, the motional current at the resonance frequency consist of both current components. For instance at a DC gate bias of $1.3 \mathrm{~V}, 3 / 4$ of the motional current is conduction modulation current while the rest arises from piezoresistive effects. The intermixing effect due to asymmetry influences the fundamental harmonic response as well as the physical nature of the electrical signal being sensed; both of which are important for understanding frequency harmonics in nanoresonators and developing efficient readout schemes for nanoscale sensors.
\end{abstract}

\section{INTRODUCTION}

Various nanomaterials such as silicon nanowires (SiNWs), graphene, carbon nanotubes (CNTs) have been used as building blocks for resonant sensing applications. The ability of these materials in exhibiting multiple electrical characteristics (piezoresistivity or conductivity modulation) results in a mechanical resonance response with multiple harmonics. This also allows for multiple transduction readout schemes such as capacitive or piezoresistive readout among others. For implementation of a selective and efficient readout scheme, in particular for nanoscale sensors, where high bandwidth and SNR can be challenging, it is crucial to know the nature of the electrical signal being sensed. For nanoresonators, most transduction schemes for mechanical resonance characterization are reported to selectively sense either the piezoresistive current or the capacitance or conductance modulation current. Here we investigate the fundamental harmonics and the selectivity of the transduction schemes for the desired component and report a qualitative and quantitative analysis of mechanical motional currents in a nanoresonator based on a single walled carbon nanotube (SWCNT).

\section{SIMULATION AND VERIFICATION}

The two used transduction schemes for carbon nanotube FET based nanoresonators as shown in Fig. 1 are piezoresistive transduction based on strain modulation and conduction modulation transduction based on field effect induced charge modulation [1]. The transduction scheme uses an RF down mixing and lock-in detection [2] with CNT as a mixer as shown in Fig. 2. This scheme requires a low bandwidth readout and minimizes the effect of parasitic capacitances.

\section{A. Piezoresistive motional current}

Piezoresistivity in nanotube arises from the strain induced resistive changes. The beam elongation as a result of out-ofplane resonance induces periodic strain $\epsilon(t)$ given by [2]

$$
\epsilon(t)=z(t)^{2} \frac{1}{2 L} \int_{0}^{L}\left(\frac{\partial \varphi(x)}{\partial x}\right)^{2} d x
$$

where $z$ is the nanotube displacement, $L$ is the length of tube and $\varphi(x)$ is the beam mode shape. The time varying resistive change $\Delta R(t)$ due to strain modulation is then given by

$$
\frac{\Delta R(t)}{R_{0}}=G_{F} \epsilon(t)
$$

where $G_{F}$ is defined as the CNT's Gauge factor [3]. The resulting piezoresistive current can then be computed by [2]

$$
i_{p}=V /\left[R_{o}+\Delta R(t)\right]
$$

$$
i_{p}=\frac{V_{s d}}{R_{0}}\left(1-z(t)^{2} G_{F} \frac{1}{2 L} \int_{0}^{L}\left(\frac{\partial \varphi(x)}{\partial x}\right)^{2} d x\right)
$$

The piezoresistive current (II term) represents a quadratic transduction scheme w.r.t CNT's displacement $z$.

\section{B. Conductance modulated motional current}

Conductance modulation due to capacitive changes have been extensively used to characterize CNT nanoresonators. The charge fluctuations due to resulting nanotube motion can be read out as a mechanical current given by [4]

$$
i_{g}=V_{s d} \frac{d G}{d V_{g}}\left(V_{g}^{a c}+z(t) \frac{V_{g}^{D C}}{C_{g}} \frac{d C_{g}}{d z}\right)
$$

where $C_{g}$ is the CNT-gate capacitance and $G$ is the CNT conductance. In contrast to piezoresistive current, the conductance modulation current (II term) exhibits a linear transduction w.r.t CNT's displacement $z$. $\left(z \ll g_{0}\right.$; CNT-gate distance)

\section{Intermixing of motional currents}

For a nanotube resonating at frequency $\omega$ with displacement amplitude $z_{o} e^{i \omega t}$, the motional currents (considering the II terms only from Eq. 4-5) can be re-written as

$$
\begin{gathered}
i_{g}=z_{o} e^{i \omega t} V_{s d} \frac{d G}{d V_{g}} \frac{V_{g}^{D C}}{C_{g}} \frac{d C_{g}}{d z}=z_{o} e^{i \omega t} K_{G} \\
i_{p}=z_{o}^{2} e^{i 2 \omega t} \frac{V_{s d}}{R_{0}} G_{F} \frac{1}{2 L} \int_{0}^{L}\left(\frac{\partial \varphi(x)}{\partial x}\right)^{2} d x=z_{o}^{2} e^{i 2 \omega t} K_{P}
\end{gathered}
$$

where $K_{G}$ and $K_{P}$ are introduced as bias dependent proportionality parameters. From Eq. 6-7, the frequency component of motional currents, $i_{g}$ and $i_{p}$, occur at $\omega$ and $2 \omega$ 
respectively and hence can be separated by two source techniques (Fig. 2). This model assumes a symmetrical displacement beam profile. However, in presence of a static bending of a beam, symmetry breaking can occur [5] and can affect the resonance behavior. In such a scenario, the nanotube displacement can be expressed as $z_{o} e^{i \omega t}=z_{s}+z_{d} e^{i \omega t}$ where $z_{s}$ and $z_{d}$ represents the static and time-varying dynamic displacement respectively. The modified expression for motional currents would then be given by

$$
\begin{gathered}
i_{g}=z_{s} K_{G}+z_{d} e^{i \omega t} K_{G} \\
i_{p}=z_{s}^{2} K_{P}+2 z_{s} z_{d} e^{i \omega t} K_{P}+z_{d}^{2} e^{i 2 \omega t} K_{P}
\end{gathered}
$$

In contrast to the symmetrical beam model, both currents are inseparable with motional current at $\omega$ consisting of terms proportional to conduction modulation and piezoresistivity, thereby hindering the selectivity of the widely employed $\omega$ transduction scheme. To study the impact of static displacement on intermixing of motional currents, we adapt the model to comprehensive CNT parameters experimentally obtained by Ning et. al. [6]; mentioned in Table I. We simulate the static and dynamic displacements and the resulting motional currents by considering electrostatic actuation on a harmonic beam resonator [4] through Simulink/MATAB.

Fig. 3 plots the conduction modulation current (Eq. 8) for various DC gate bias exhibiting spring hardening effect and increase in resonance current (Inset-Fig. 3) at resonance frequency $\omega$. The higher order derivatives such as $d^{2} C_{g} / d z^{2}$ lead to a negligible current at $2 \omega$. In contrast, the piezoresistive current in Fig. 4 has components at both $\omega$ and $2 \omega$ as expected from Eq. 9. While the $2 \omega$ component of total motional current (Eq. $8+$ Eq. 9) is purely piezoresistive, $\omega$ component results from mixing of both currents as shown in Fig. 5. For the considered set of CNT model parameters, the intermixing effect increases the peak resonance signal as well as hinders the selectivity of the $\omega$ measurement technique.

\section{EXPERIMENTS AND RESULTS}

For experimental investigation of the intermixing effect, we use a small bandgap semiconducting (SGS) nanotube resonator device (Fig. 6) as they exhibit both gate dependent conductance modulation and high Gauge factor in contrast to purely semiconducting or metallic nanotubes [3]. We characterize the mechanical behavior of the nanotube by both $\omega$ and $2 \omega$ transduction at an RF down mixing frequency $\Delta \omega=10 \mathrm{kHz}$ exhibiting a resonance frequency at $72.6 \mathrm{MHz}$ (Fig. 6f) at room temperature $(300 \mathrm{~K})$ and low vacuum $\left(<10^{-3} \mathrm{mbar}\right)$. The measured high resonance frequency in comparison to theoretical eigenmode suggest a slack-free CNT resonator. The Lorentzian fit to measured frequency spectral responses was used to extract the peak resonance current.

Fig. 7 shows purely piezoresistive current at various DC gate bias detected through $2 \omega$ scheme. To quantity the currents, the dependency $z_{d}^{2} \propto\left(V_{g}^{D C}\right)^{2}$ was used for power fitting. Peak resonance currents obtained from $\omega$ scheme as shown in Fig. 8 were also fitted, with an additional dependency $z_{s} \propto\left(V_{g}^{D C}\right)^{2}$, to separate the motional currents. To account for the intermixing effect, square and fourth power fit was used (the peak current was normalized to background current, I term of Eq. 5, to account for bias dependent conductance). The proportionality parameter $K_{P}$ obtained with power fits from $\omega$ and $2 \omega$ measurements were extracted to be 0.268 and 0.263 respectively supporting the validity of fits and intermixing between motional currents. In addition, the term $d G / d V_{g}$ extracted from $\mathrm{K}_{\mathrm{G}}, 4.33 \times 10^{-7} \mathrm{~S} / \mathrm{V}$ and CNT-FET $\mathrm{I}_{\mathrm{D}}-\mathrm{V}_{\mathrm{G}}$ transfer characteristic, $3.58 \times 10^{-7} \mathrm{~S} / \mathrm{V}$ shows a good agreement from the fit based on intermixing effect.

\section{DISCUSSION AND CONCLUSION}

With the extracted parameters, we quantify the intermixing effect at resonance frequency $\omega$ for the measured nanotube device. As shown in Fig. 9, for low DC gate bias of $0.5 \mathrm{~V}$, piezoresistive current constitutes only an estimated $4 \%$ of the total motional current. This increases to $26 \%$ while the remaining $74 \%$ arises from conduction modulation current at a bias of $1.4 \mathrm{~V}$ due to asymmetrical beam profile as a result of DC electrostatic force. Amplitude and Q-factor fluctuations, observed in nanoresonators, could lead to inaccuracies in the estimated current components. Piezoresistivity due to asymmetrical beam profile has also been observed in a $90 \mathrm{~nm}$ thick silicon nanowire resonator [2], however at a high gate bias of $15 \mathrm{~V}$, suggesting the significance of intermixing in subnanometer resonators at low bias; especially in non-linear resonators operating at higher gate bias resulting in several harmonics. Differences in resonator characteristics like Gauge factor, chirality or conductance such as the one considered in Table I can also lead to an intermixing with $50-50 \%$ contribution, thereby effecting the physical nature of the electrical signal and the selectivity of $\omega$ transduction scheme, both of which are important for understanding the harmonic components of resonators and in the design of highly efficient and sensitive readout schemes for nanoscale sensors.

\section{ACKNOWLEDGMENT}

The authors acknowledge the support of cleanroom facility at Binnig and Rohrer Nanotechnology Center $(B R N C)$ at IBM Rüschlikon and FIRST CLA at ETH Zurich. We would also like to thank the Swiss National Science Foundation (SNSFProject No. 153292) for the financial support.

\section{REFERENCES}

[1] S. W. Lee, S. Truax, L. Yu, C. Roman and C. Hierold, "Carbon nanotube resonators with capacitive and piezoresistive current modulation readout," Appl. Phys. Lett., 103, 033117, 2013.

[2] M. Sansa, M. F. Regulez, J. Llobet, A. S. Paulo and F. P. Murano, "Highsensitivity linear piezoresistive transduction for nanomechanical beam resonators," Nat. Comm., 5, 4313, 2013.

[3] W. Obitayo and T. Liu, "A Review: Carbon nanotube based piezoresistive strain sensors," J. Sensors, 652438, 2012.

[4] V. Sazonova, Y. Yaish, H. Üstünel, D. Roundy, T. A. Arias and P. L. McEuen, "A tunable carbon nanotube electromechanical oscillator,"Nature, 431, 2004, pp 284-287.

[5] A. Eichler, J. Moser, M. I. Dykman and A. Bachtold, "Symmetry breaking in a mechanical resonator made from a carbon nanotube," Nat. Comm., 4, 2843, 2013.

[6] Z. Y. Ning, M. Q. Fu, T. W. Shi, Y. Guo, X. L. Wei, S. Gao and Q. Chen, "In situ multiproperty measurements of individual nanomaterials in SEM and correlation with their atomic structures," Nanotechnology, 25, $275703,2014$. 


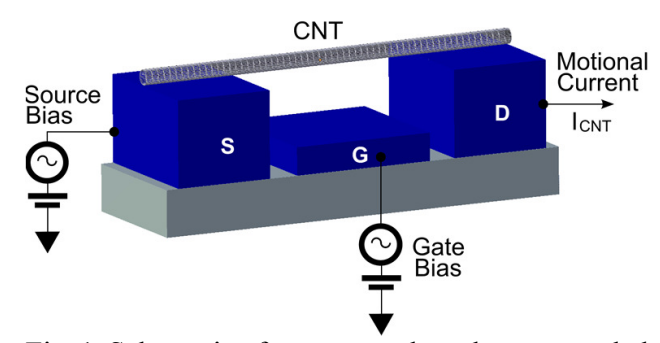

Fig. 1. Schematic of a resonator based on suspended carbon nanotube between source $(S)$ and drain $(D)$ electrode of a field effect transistor with bottom gate $(\mathrm{G})$ architecture.

\begin{tabular}{|l|l|l|}
\hline \multicolumn{1}{|c|}{ CNT parameter } & \multicolumn{1}{c|}{ Value } & \multicolumn{1}{c|}{ Unit } \\
\hline Radius $(\mathrm{r})$ & 1.1 & $\mathrm{~nm}$ \\
\hline Length $(\mathrm{L})$ & 1.59 & $\mu \mathrm{m}$ \\
\hline Density $(\rho)$ & 1400 & $\mathrm{~kg} / \mathrm{m}^{3}$ \\
\hline Mass $(\mathrm{m})$ & $2.54 \times 10^{-21}$ & $\mathrm{~kg}$ \\
\hline Cross-sectional area $\left(\mathrm{A}_{\mathrm{c}}\right)$ & $2.29 \times 10^{-18}$ & $\mathrm{~m}^{2}$ \\
\hline Young's modulus $(\mathrm{E})$ & 154 & $\mathrm{GPa}$ \\
\hline Device resistance $\left(\mathrm{R}_{0}\right)$ & 1.01 & $\mathrm{Mohm}$ \\
\hline CNT-gate distance $\left(\mathrm{g}_{0}\right)$ & 300 & $\mathrm{~nm}$ \\
\hline Zero-strain bandgap $\left(\mathrm{E}_{0}\right)$ & 113.2649 & $\mathrm{meV}$ \\
\hline dE/d $\epsilon$ & 49.8 & $\mathrm{meV} / \%$ \\
\hline dG/dV & $1.43 \times 10^{-7}$ & $\mathrm{~S} / \mathrm{V}$ \\
\hline Spring constant & $1.53 \times 10^{-4}$ & $\mathrm{~N} / \mathrm{m}$ \\
\hline Damping factor $(\mathrm{b})$ & $9.7 \times 10^{-15}$ & $\mathrm{~kg} / \mathrm{s}$ \\
\hline Source bias $\left(\mathrm{V}_{\mathrm{sd}}{ }^{\mathrm{AC}}, \mathrm{V}_{\mathrm{sd}}{ }^{\mathrm{DC}}\right)$ & 10 & $\mathrm{mV}$ \\
\hline Gate $\mathrm{AC}$ bias $\left(\mathrm{V}_{\mathrm{g}}{ }^{\mathrm{AC}}\right)$ & 10 & $\mathrm{mV}$ \\
\hline
\end{tabular}

Table. I. CNT parameters experimentally extracted by Ning et.al.[6] through resonance frequency measurement, DC measurements, Raman spectroscopy, scanning electron microscopy (SEM), and transmission electron microscopy (TEM).

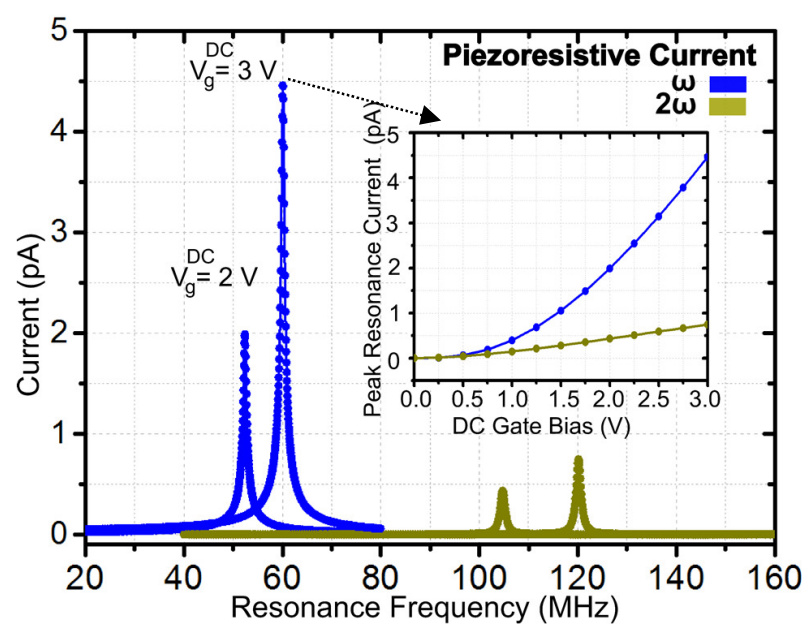

Fig. 4. Piezoresistive current; Eq. 9 simulated for various DC gate bias for CNT resonator at $\omega$ and $2 \omega$ due to $2 z_{s} z_{d}$ and $z_{d}^{2}$ respectively. Inset - Peak resonance current vs DC gate bias.

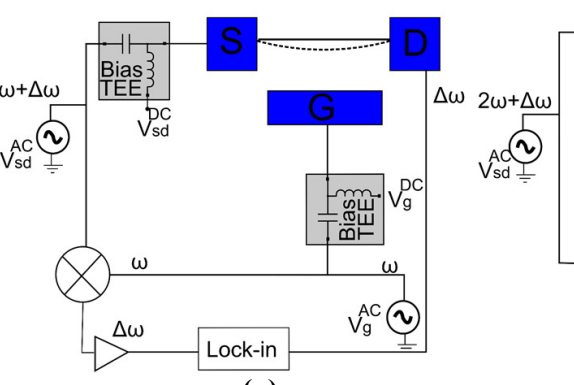

(a)

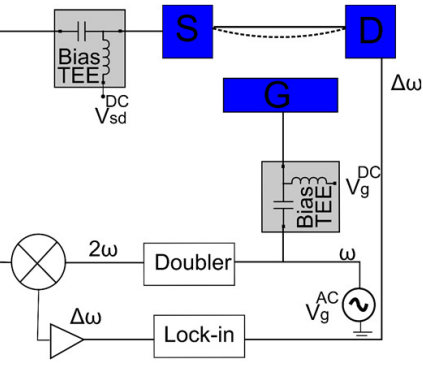

(b)
Fig. 2. (a) Experimental setup using RF mixing and lock-in technique for readout of motional current components at resonance frequency $\omega$; (b) Modified setup to readout the current component at $2 \omega$.

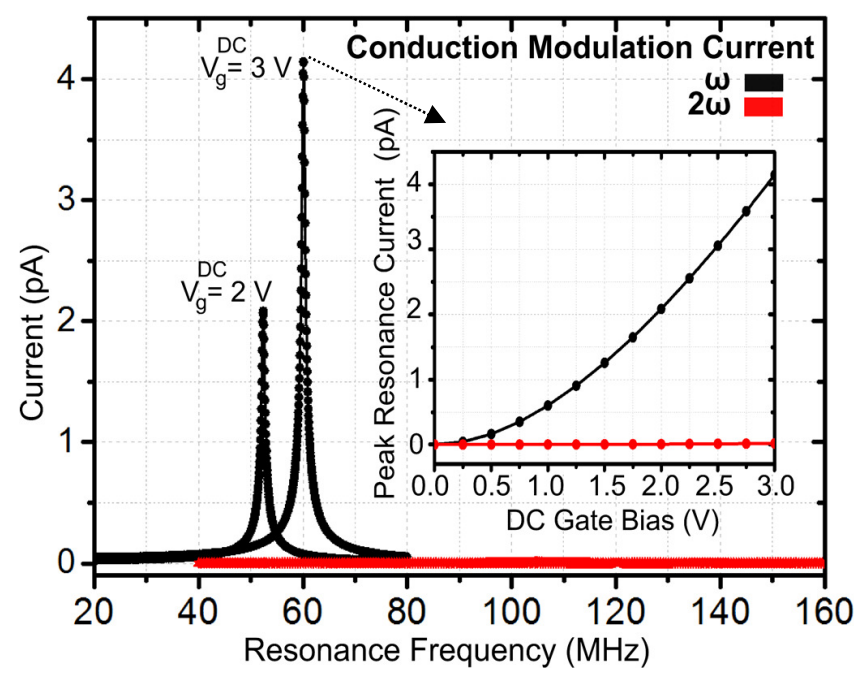

Fig. 3. Conduction modulation current; Eq. 8 simulated for various DC gate bias for CNT resonator at $\omega$. The higher second order terms $\left(\mathrm{d}^{2} \mathrm{C} / \mathrm{dz}^{2}\right)$ results in a negligible $2 \omega$ component. Inset - Peak resonance current vs DC gate bias.

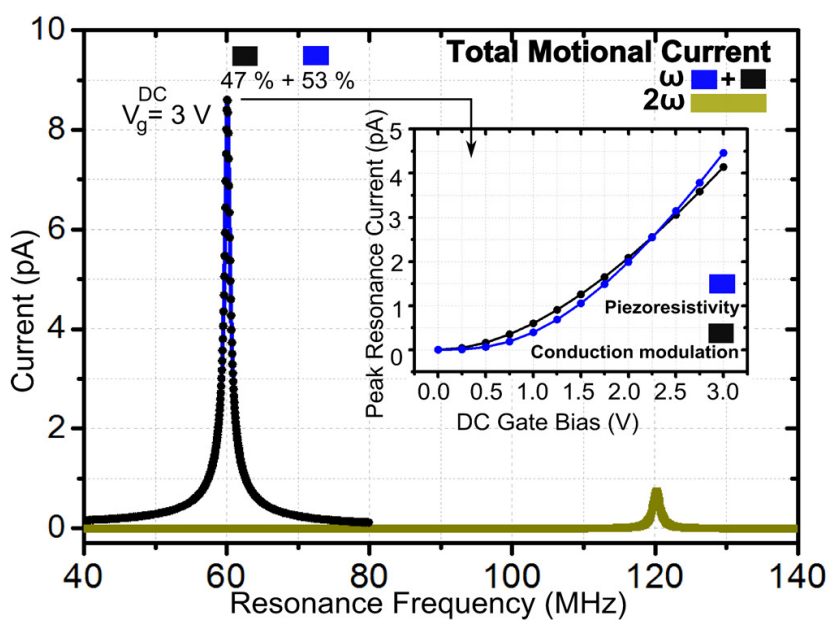

Fig. 5. Total motional current (Eq. 8+Eq. 9) at $3 \mathrm{~V}$ DC gate bias for CNT resonator at $\omega$ and $2 \omega$. While $2 \omega$ is purely piezoresistive, the current at $\omega$ consist of $47 \%$ conduction modulation current and $53 \%$ piezoresistive current (extracted from peak currents -Inset) 


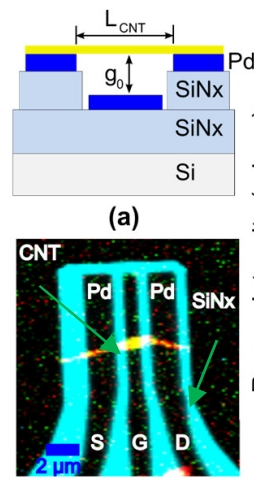

(b)

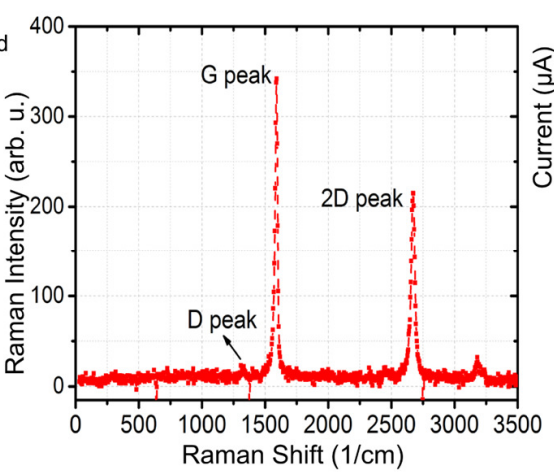

(c)

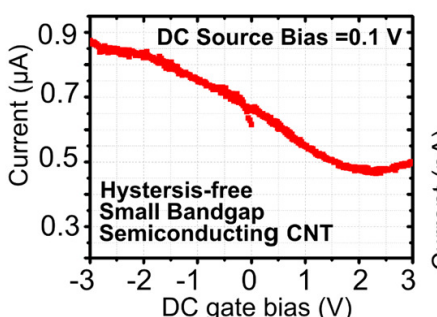

(d)

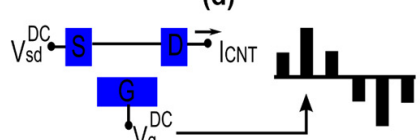

(e)

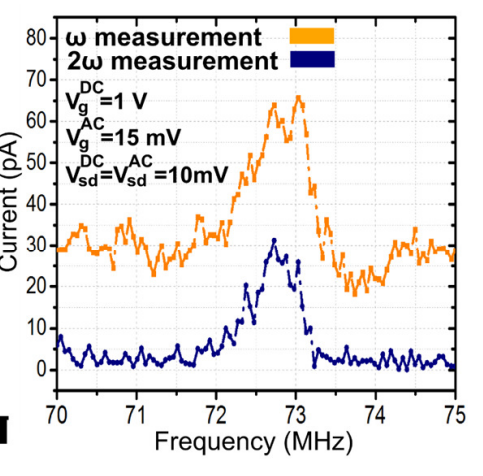

(f)

Fig. 6. (a) Device architecture of the CNT-FET based resonator with suspended channel length of $2 \mu \mathrm{m}$ and CNT-gate distance of $275 \mathrm{~nm}$. (b) Raman spectroscopy of the fabricated device with a nanotube obtained after dry-transfer process; (c) Raman spectrum measured for the suspended nanotube; (d) DC CNT-FET transfer characteristics exhibiting a small bandgap semiconducting (SGS) behavior; (e) Pulsed gate DC measurement for extracting hysteresis free performance; (f) Resonance frequency characterization through two source $\omega$ and $2 \omega$ detection technique. Both scheme detects the resonance frequency at $72.6 \mathrm{MHz}$ with different motional current amplitudes.

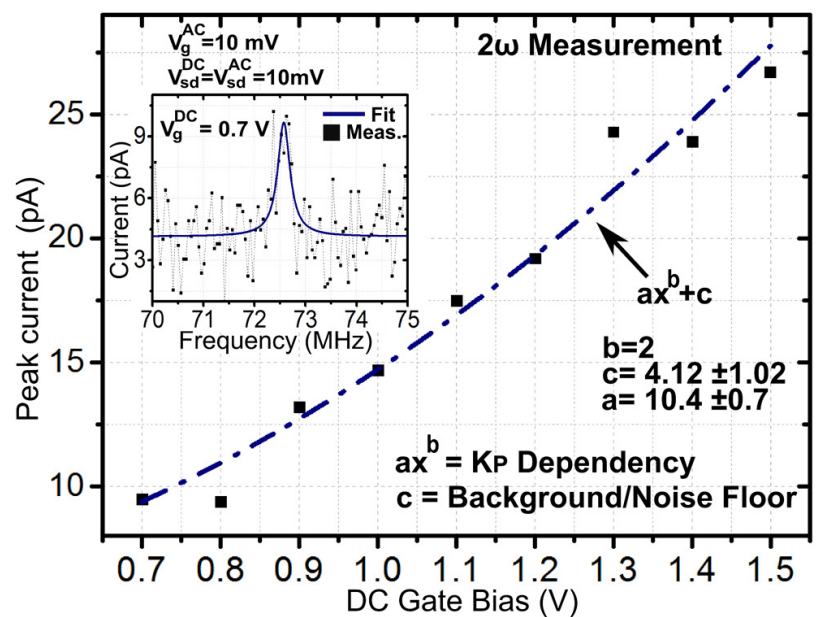

Fig. 7. Resonance peak current vs DC gate bias for $2 \omega$ measurement corresponding to piezoresistive current. Inset - Measured frequency response with Lorentzian fit to extract parameters.

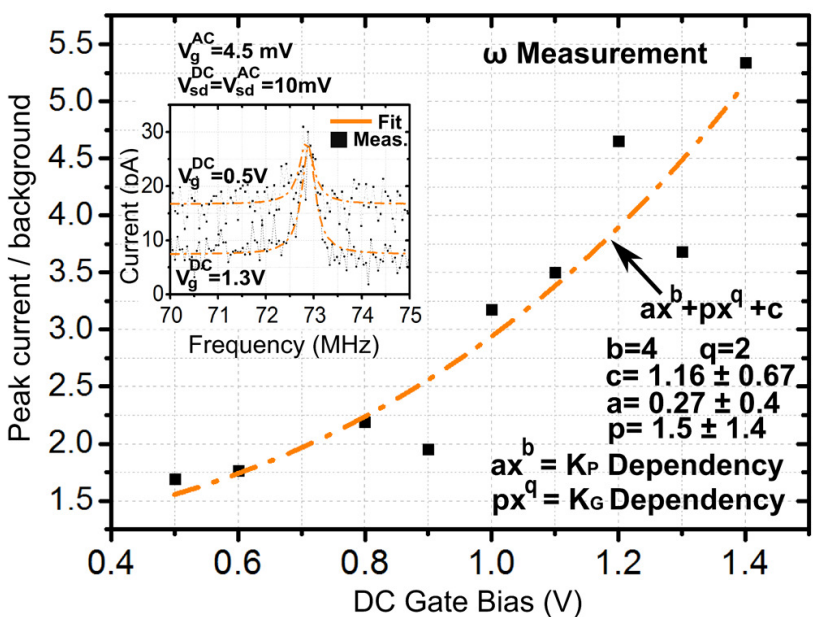

Fig. 8. Resonance peak current (normalized to background current$\mathrm{dG} / \mathrm{dV}$ dependency) vs DC gate bias for $\omega$ measurement corresponding to conduction modulation and piezoresistive current. Inset - Measured frequency response with Lorentzian fit.

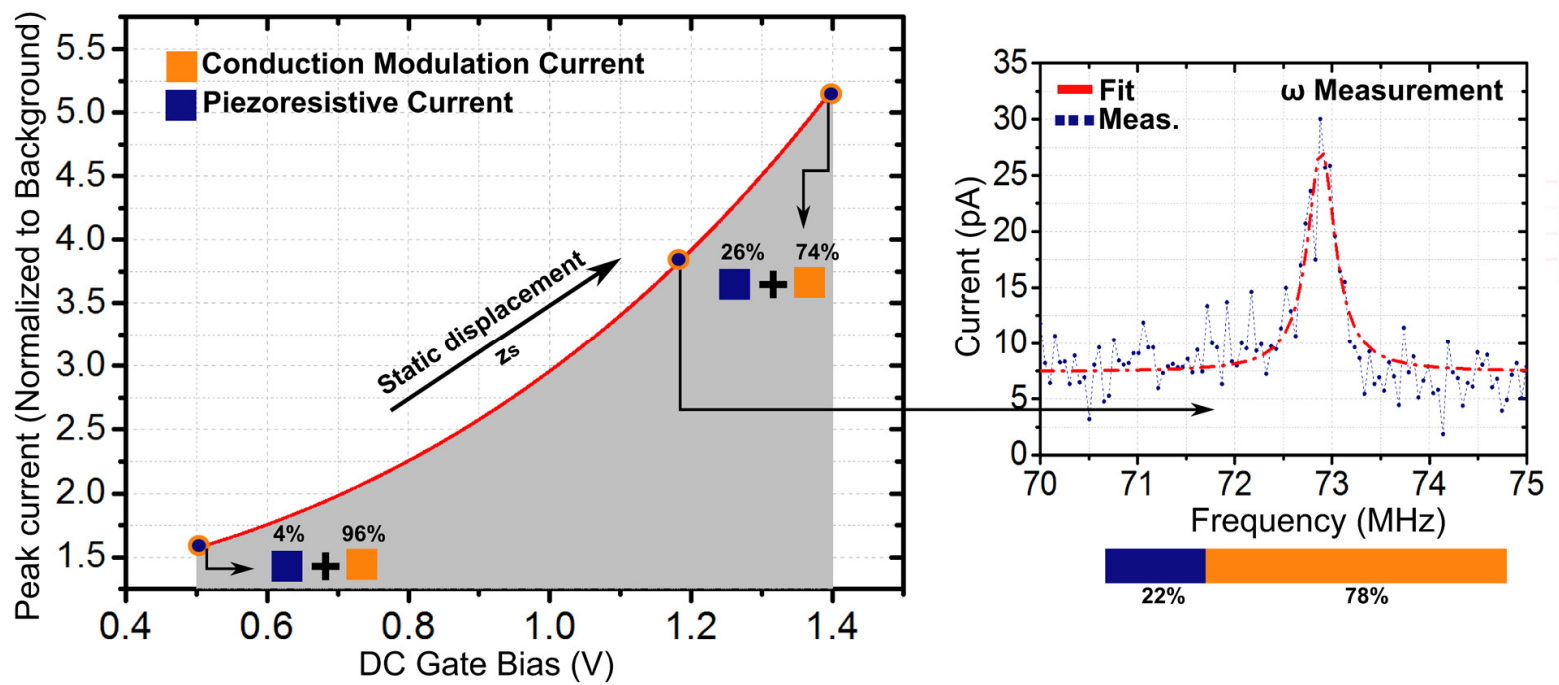

Fig. 9. Strength of intermixing of piezoresistive current and conduction modulation current as a function of DC gate bias. The increase in the nanotube's static displacement is estimated to increase the intermixing component $2 z_{S} z_{d} K_{P}$ to one-fourth of the total motional current. 